\title{
Rheology of cement paste under high pressure
}

\author{
Jae Hong Kim ${ }^{\text {a }}$, Seung Hee Kwon ${ }^{\text {b, }}{ }^{*}$, Shiho Kawashima ${ }^{c}$, Hong Jae Yim ${ }^{\text {d }}$ \\ a School of Urban and Environmental Engineering, Ulsan National Institute of Science and Technology, Republic of Korea \\ ${ }^{\mathrm{b}}$ Department of Civil and Environmental Engineering, Myongji University, Republic of Korea \\ ${ }^{\mathrm{c}}$ Department of Civil Engineering and Engineering Mechanics, Columbia University, USA \\ ${ }^{\mathrm{d}}$ Department of Construction and Disaster Prevention Engineering, Kyungpook National University, Republic of Korea
}

\section{A R T I C L E I N F O}

\section{Article history:}

Received 7 April 2015

Received in revised form

22 September 2016

Accepted 21 November 2016

Available online 22 November 2016

\section{Keywords:}

Cement paste

Pumping

Rheology

Characterization

Pressure

Viscosity

\begin{abstract}
A B S T R A C T
The objective of this study was to investigate whether cementitious materials undergo changes during pumping processes due to pressure variation. The influence of pressure on the rheological properties of cement pastes, which assumably represented the lubricating layer that forms along the profile of concrete during pumping, was evaluated using a rotational rheometer with a high-pressure cell. Cement pastes with water-to-cement ratios ranging from 0.35 to 0.6 were tested according to a protocol designed to simulate the conditions of an actual pumping process based on field tests. The shear rates, shearing durations, and pressure levels from 0 to $30 \mathrm{MPa}$ were experimentally simulated. The test results indicated that below a certain water-to-cement ratio (0.40) elevated pressures lead to changes in the rheological properties, while changes were negligible when the ratio was above this threshold. Further, at low water-to-cement ratios the thixotropy of the cement paste can reverse into rheopexy after pressurization.
\end{abstract}

() 2016 Elsevier Ltd. All rights reserved.

\section{Introduction}

Pumping is a general way to transport cement-based materials at construction sites [1,2]. Such pumped mixtures are subjected to high pressure during pumping, especially in large-scale construction such as high-rise buildings, long-span bridges, long-distance tunnels, oil-well cementing applications, and ground strengthening by injection of paste or mortar [3-6].

Research on blockage during pumping [8] and the relationship between pumping flow and pressure [9] confirmed that a lubricating layer is formed near the inner surface of the pipe [7]. The development of the lubricating layer is caused by shear-induced particle migration. The phenomenon refers to in which particles in a suspension move into the region with a lower rate of shear strain. When a concrete mix flows in a pipe, the velocity is at its maximum at the center of the cross section and decreases as it approaches the inner surface of the pipe. The rate of shear strain inversely follows the velocity profile along the cross section. As a result, coarse and fine aggregates concentrate toward the center and a portion of the cement paste phase forms a lubricating layer.

\footnotetext{
* Corresponding author. 116, Myongji-ro, Cheoin-gu, Yongin, Gyeonggi-do 449728, South Korea.

E-mail address: kwon08@mju.ac.kr (S.H. Kwon).
}

The thickness of the lubricating layer is in the range of 1 to $5 \mathrm{~mm}$, which was confirmed by experiments $[10,11]$ and theoretical evaluations [12].

The rheological properties of the lubricating layer determine the flow-rate of the pumped concrete, while those of the bulk concrete mix are less influential (see Fig. 1). The discharge rate of the pumped concrete highly depends on the lubricating layer. The conventional vane-type viscometer is acceptable for measuring the viscosity of concrete, but it is hard to develop and investigate the lubricating layer that forms at the interface between the pipe surface and the concrete. Tribometers have been developed to evaluate the rheological properties of the lubricating layer [3,9,13], which replaces a rotating vane geometry with a cylinder. The surface of the cylinder mimics the inner boundary of a pipe, and the lubricating layer is expected to develop near its surface. The formulated models accompanying the tribometer were able to estimate the relationship between the pumping pressure and the discharge rate in the unit of $\mathrm{m}^{3} / \mathrm{h}[4,9,23]$.

On the other hand, several hypothetical mechanisms have been suggested for how pressure can change the rheological properties of the lubricating layer and subsequently affect the pumping efficiency. The first hypothesis is that dispersion of cement particles increases under high pressure. Their dispersion reduces the interparticle distance causing to increase their attraction potential, 
which results in the fluidity loss of their suspension. From this perspective, the total surface area of the cement particles consequently increases with increasing pumping pressure and leaves the mix with an insufficient high-range water-reducing admixture (HRWRA) dosage to maintain its flow [17]. The second potential mechanism is that the length of the side-chains of the polycarboxylate-based HRWRA might be reduced or cut with increasing pressure [18]. A third potential mechanism is that the sand and coarse aggregate absorb more free water or HRWRA under pressure [1]. Each of the three hypothetical mechanisms addresses the increase in the rheological properties observed during pumping. Even though field tests have been performed to investigate the efficiency of pumping pressure and flow rate, the actual mechanism has not yet been verified. One of the challenges for the verification is related to the difficulty in quantitatively measuring the rheological properties of cement-based materials under high pressure and shearing.

In this study, the first hypothesis was tested: The effect of pressure on the rheology of the cement paste consisting of the lubricating layer in pumped concrete. Other influencing factors, such as temperature, aggregate absorption, and admixture compatibility, were excluded to avoid their coupling effects on the rheology under a high pressure. A special rheometer was used to measure rheological properties of a sample under various hydrostatic pressure levels. The measured geometry was intended to evaluate the lubricating layer. For this purpose, firstly it was necessary to determine the appropriate range of shear rates to apply so that they were representative of the shear conditions the lubricating layer experiences during concrete pumping. A protocol specifying the shear rate range of interest was defined by comparing previously measured field test results of concretes with a Newtonian fluid model. From there, cement paste samples with various water-to-cement $(w / \mathrm{cm})$ ratios ranging from 0.35 to 0.60 were tested. The range of applied pressure was up to $30 \mathrm{MPa}$ (300 bar), which covers most general cases for construction applications.

\section{Experiment}

\subsection{Sample preparation}

Cement paste samples were prepared using ordinary Type I Portland cement. The specific gravity of the cement was 3.14, and the Blaine was $335 \mathrm{~kg} / \mathrm{m}^{2}$. Both values were measured following standard test methods (ASTM C 188-09 and ASTM C 204-11, respectively). Table 1 shows the mix proportion used to prepare the paste samples with a batch size of approximately $50 \mathrm{~mL}$. The solid volume fraction, $\phi$, in the table was also calculated using the specific gravity of the cement. The temperature $\left(21^{\circ} \mathrm{C}\right)$ and humidity (50\%) were maintained continuously throughout the following experiment.

The mixing protocol for all samples was kept constant: (1) 0.5 min hand mixing; (2) 2.5 min scraping; and (3) 0.5 min additional hand mixing. The total mixing time was $3.5 \mathrm{~min}$. All samples were prepared in a $150 \mathrm{~mm}$-beaker and subjected to mixing with a glass rod at approximately 1 rps.

\subsection{Instrument and sample loading}

Neat cement paste samples were tested to measure their rheological properties under high pressure. The measured geometry to investigate the lubricating layer is very similar to a conventional concentric cylinder, as shown in Fig. 2. The radii of the inner and outer cylinders were 17.5 and $19.5 \mathrm{~mm}$, respectively. The gap size for the Couette flow was $2 \mathrm{~mm}$, which was sufficiently larger than the maximum size of the cement particles.

In order to apply pressure to the sample, the inner space of the cup cylinder was fully filled with paraffin oil and the cell was tightly capped with high-strength bolts after the sample was loaded inside. The paraffin oil, which has relatively low viscosity and low water-solubility, was continuously added into the sealed cylinder using a hand pump. The inner cylinder was physically disconnected from the outer rotor but had an axis of rotation. A magnetic holder was needed to transfer the rotation torque to the inner cylinder (mounted on a sapphire bearing) - through this magnetic coupling shear was applied to the sample. Fig. 3 shows the pressure cell loaded into a rheometer.

The relatively small gap size of the coaxial cylinders made it difficult to load some of the samples. The cement pastes proportioned with $\mathrm{w} / \mathrm{cm}$ higher than 0.45 showed enough fluidity to permeate the gap space, where a small funnel was used. For cement pastes with a lower $\mathrm{w} / \mathrm{cm}$, the small spaces were more difficult to

Table 1

Mix proportions for $50 \mathrm{~mL}$ cement paste samples.

\begin{tabular}{llll}
\hline$w / c m$ & $\phi$ & Water, g & Cement, g \\
\hline 0.35 & 0.48 & 26 & 75 \\
0.40 & 0.44 & 28 & 70 \\
0.45 & 0.41 & 29 & 65 \\
0.50 & 0.39 & 31 & 61 \\
0.60 & 0.35 & 33 & 54 \\
\hline
\end{tabular}

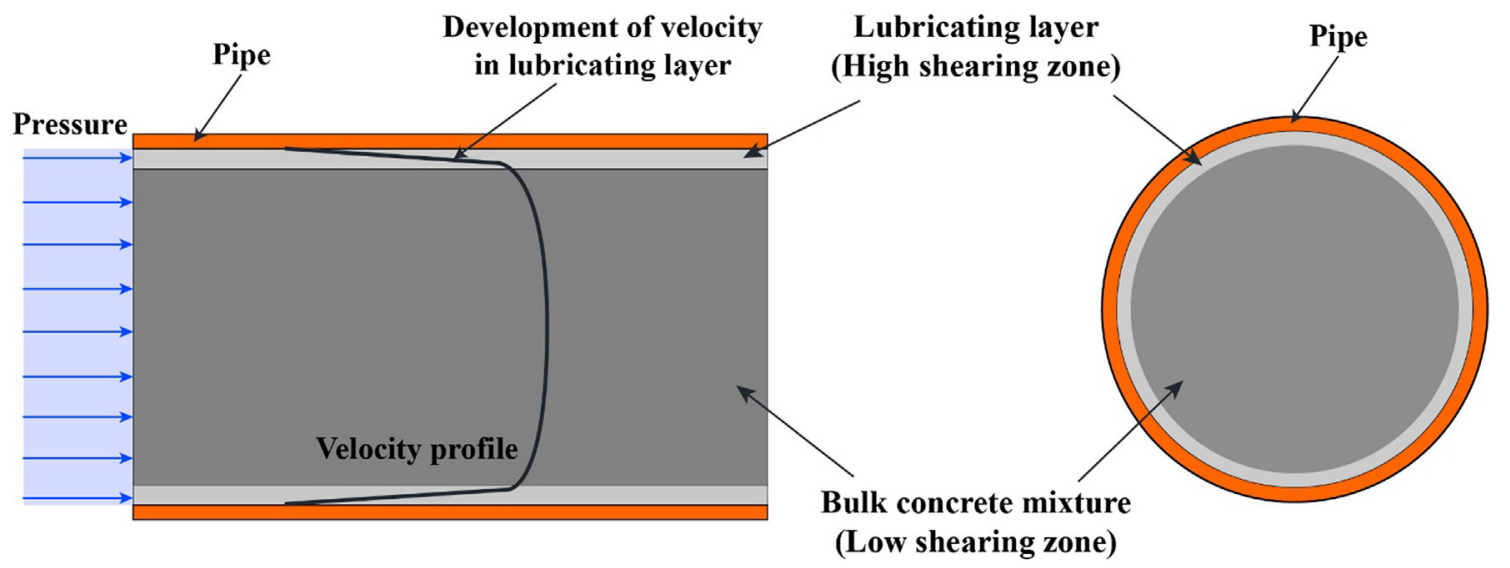

Fig. 1. Schematic description of pipe flow of pumped concrete. 
Table 2

Summary of the mockup pumping test.

\begin{tabular}{|c|c|c|c|c|}
\hline \multirow[t]{2}{*}{ Pipe length } & \multicolumn{2}{|l|}{$350 \mathrm{~m}$} & \multicolumn{2}{|l|}{$548 \mathrm{~m}$} \\
\hline & Slowest & Fastest & Slowest & Fastest \\
\hline Flow rate, $\mathrm{m}^{3} / \mathrm{h}$ & 13 & 62 & 9 & 45 \\
\hline Pressure gradient, kPa/m & 25 & 28 & 15 & 25 \\
\hline Peak pressure at the mouth, $\mathrm{MPa}$ & 8.75 & 9.80 & 8.22 & 13.7 \\
\hline Plastic viscosity of bulk concrete, Pa.s & 152 & 23 & 144 & 47.1 \\
\hline Plastic viscosity of the lubricating layer, $\mathrm{Pa} \cdot \mathrm{s}$ & 4.54 & 1.48 & 3.99 & 1.58 \\
\hline Viscosity by Eq. (3), Pa.s & 44 & 11 & 39 & 13 \\
\hline Max shear rate by Eq. (4), $\mathrm{s}^{-1}$ & 9 & 43 & 6 & 31 \\
\hline Max shear rate in the lubricating layer, $\mathrm{s}^{-1}$ & 302 & 660 & 217 & 924 \\
\hline
\end{tabular}

fill. For this reason, the paste was first spread on the inner cylinder before being placed in the outer cylinder. Vibration and tapping was applied to consolidate, then the rest of the paste sample was added to fill the gap space. The time for loading a sample and closing the high-pressure cell was approximately $10 \mathrm{~min}$ after the end of mixing. Therefore, the rheological test, which is introduced in section 2.4, was set to start at $13.5 \mathrm{~min}$.

\subsection{Shear-rate ranges of interest}

The development of the lubricating layer changes the distribution of the rate of shear strain, as shown in Fig. 1. Finding a link between the bulk concrete and the lubricating layer allows us to determine the range of shear rates that should be tested. Assuming that a concrete is a Newtonian fluid, the Navier-Stokes equation for an incompressible viscous fluid is given by

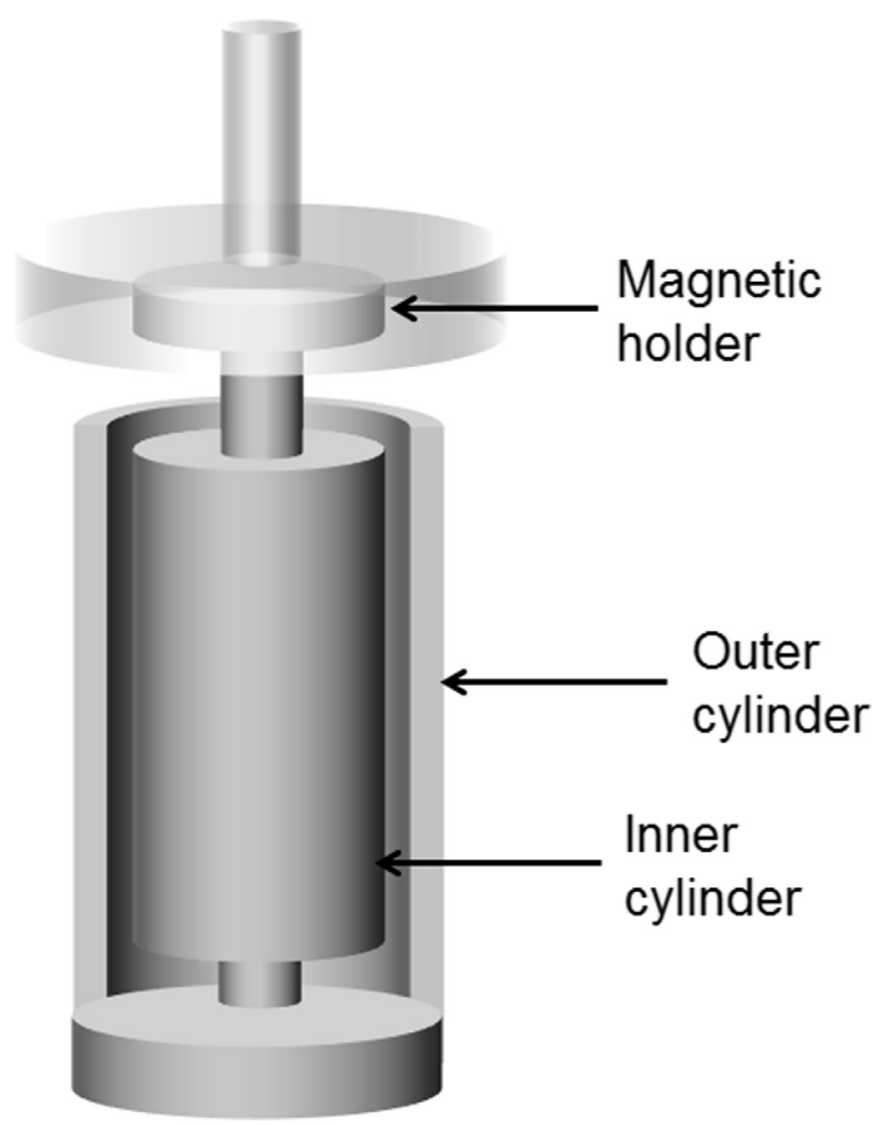

Fig. 2. Schematic diagram of a high-pressure cell. $\rho \frac{D \vec{v}}{D t}=-\vec{\nabla} p+\eta \nabla^{2} v$

where $v$ and $p$ are the fluid velocity and pressure, respectively. The velocity field is governed by the viscosity $\eta$ and the density $\rho$. The Poiseuille flow simplifies the flow in the pipeline to a steady confined flow under a pressure gradient. Solving the boundary value problem using the cylindrical coordinate gives the altitudinal velocity at a radial distance $(r)$ by

$v_{z}(r)=-\frac{1}{4 \eta} \frac{\partial p}{\partial z}\left(\frac{D^{2}}{4}-r^{2}\right)$

where $D$ is the diameter of a pipe and $\partial p / \partial z$ is the pressure gradient in the longitudinal direction. The flow rate through the pipe is then calculated by integrating the velocity over the cross-sectional area,

$\mathrm{Q}=\int v_{z} d A=-\frac{\pi}{128} \frac{D^{4}}{\eta} \frac{\partial p}{\partial z}$

The rate of shear strain is obtained by differentiating the velocity,

$\dot{\gamma}(r)=\frac{\partial v_{z}}{\partial r}=\frac{r}{2 \eta} \frac{\partial p}{\partial z}$

The maximum shear rate is reached at the inner surface of the pipe $(r=D / 2)$ and the shear rate vanishes at the center of the pipe $(r=0)$.

As an example calculation considered a mockup test performed

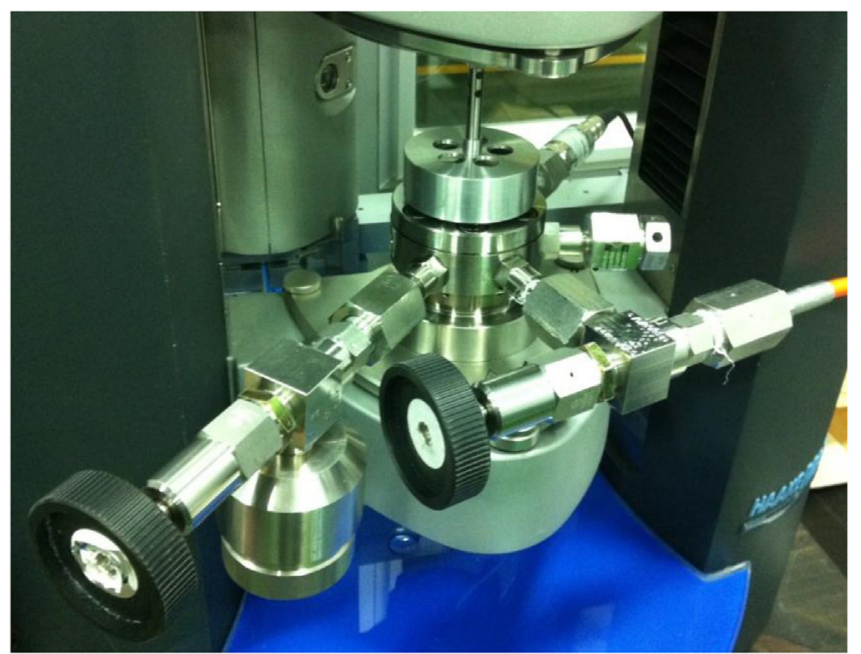

Fig. 3. High-pressure cell in a rheometer. 
in a previous study [4]. Table 2 reports selected results. For the slowest pumping case through a $350 \mathrm{~m}$ long 127-mm diameter pipeline, Eq. (3) gives the viscosity of the concrete mix: $44 \mathrm{~Pa}$ s with $Q=13 \mathrm{~m}^{3} / \mathrm{h}$ and $\partial p / \partial z=25 \mathrm{kPa} / \mathrm{m}$. The maximum shear rate is then given by Eq. (4): $9 \mathrm{~s}^{-1}$ at $r=D / 2$. Comparing the rheological measurements of the bulk concrete mix with that of the surrounding lubricating layer allowed us to further estimate the shear rate experienced in the lubricating layer. The plastic viscosities of the bulk concrete and the lubricating layer were 152 and $4.54 \mathrm{~Pa} \mathrm{~s}$, respectively. The plastic viscosity of the bulk concrete was 33.5 times greater than that of the lubricating layer. Eq. (4) again indicates that the maximum shear rate is inversely proportional to the viscosity. Therefore, the maximum shear rate in the lubricating layer can be calculated by applying the same scaling factor, giving $302 \mathrm{~s}^{-1}$ for the slowest pumping case. The calculated maximum shear rate for the fastest and $548 \mathrm{~m}$ long pumping cases are also listed in Table 2.

Thus, a test protocol for rheological measurement should apply a shear rate up to $1,000 \mathrm{~s}^{-1}$ to take the pumping process into consideration. Note that gravity-induced flow, such as a slump flow, is reportedly on the order of $10 s^{-1}$ [19]. Interest in relatively high rates of shear strain motivated us to measure the flow curve in the range of 10 to $1,200 \mathrm{~s}^{-1}$, which is the highest value attainable by the rheometer. In addition, the peak pressure level in the field-test results was up to $13.7 \mathrm{MPa}$, as reported in Table 2, and so the maximum pressure for the test was set to $30 \mathrm{MPa}$.

\subsection{Rheological measurement}

The shear stress was recorded in 10 linear-scale steps, where each step was $20 \mathrm{~s}$ for a designated rate of shear strain. For each measurement, two flow curves were obtained: up-curves and down-curves. Fig. 4 shows the protocol for each measurement, which was taken over $400 \mathrm{~s}$. To illustrate a typical measurement, Fig. 5(a) shows the flow curve of a cement paste prepared with $w /$ $\mathrm{cm}=0.35$. The blue and red curves were the up-curve and the down-curve, respectively, obtained during Measurement 1 . Shear thinning behavior, which is common in cement paste, placed the first point (recorded at $10 \mathrm{~s}^{-1}$ ) lower than a linear trend. Ignoring the first point allowed us to fit the measurement with a Bingham model. Two flow curves at each measurement were then parametrized by

$\tau(\dot{\gamma})=\tau_{y}+\eta_{p} \dot{\gamma}$

where $\tau_{y}$ is the yield stress and $\eta_{p}$ is the plastic viscosity. The model was developed in the range of 133 to $1,200 \mathrm{~s}^{-1}$. At such a high rate, the shear thinning was very weak (as can be seen in Fig. 5).

The area between the up- and down-curves indicates the level of thixotropy of a sample [20]. Integrating from ' 0 ' to the max shear rate $\left(\gamma_{\max }=1,200 \mathrm{~s}^{-1}\right)$ gives the area under the flow curve, which is the rate of dissipated energy density:

$\dot{e}=\int \tau d \dot{\gamma}=\tau_{y} \dot{\gamma}_{\max }+\frac{\eta_{p}}{2} \dot{\gamma}_{\max }^{2}$

The thixotropic parameter $\left(A_{t h}\right)$ of a sample is defined as the difference in the energy rate.

$A_{t h}=\dot{\gamma}_{\max } \Delta \tau_{y}+\frac{\dot{\gamma}_{\max }^{2}}{2} \Delta \eta_{p}$

For a thixotropic fluid, the up-curve has higher yield stress and lower plastic viscosity thanss the down-curve. Therefore, for a thixotropic material such as cement paste, the thixotropic parameter should yield a positive value. This parameter can give a relative comparison of each mix rather than a material property, so its value is not consistent when the range of shear rates is changed. Finally, each measurement gives five items of output data: yield stress of the up-curve, plastic viscosity of the up-curve, stress of the downcurve, viscosity of the down-curve, and the thixotropic parameter.

Fig. 5 shows the timetable for a test run. Each sample was tested to obtain three rheological measurements, which will hereafter be referred to as Measurements 1, 2, and 3. Changes in the rheological properties of a single sample were measured through the three steps. Mixing was done for $3.5 \mathrm{~min}$, as previously stated, and the loading of the sample into the pressure cell took about $10 \mathrm{~min}$. Measurement 1 was performed at atmospheric pressure. That is the reference value of the sample. The internal pressure of the cell was subsequently increased using a hydraulic hand pump, which was set within approximately $15 \mathrm{~min}$. Measurement 2 was made under the specified high pressure. Measurement 3 was performed under high pressure after 4-min of high shear agitation at $1,200 \mathrm{~s}^{-1}$.

\section{Results and discussion}

\subsection{Verification on the range of shear rate}

Fig. 6 shows the measurement of a cement paste sample with $w /$ $\mathrm{cm}=0.35$, where a clear Bingham fluid-like behavior can be found in both measurements. For instance, the parameters defined in the previous section for Measurement 1 (Fig. 6(a)) are as follows: $\tau_{y}=187 \mathrm{~Pa}$ and $\eta_{p}=0.20 \mathrm{~Pa} \cdot \mathrm{s}$ on the up-curve; $\tau_{y}=115 \mathrm{~Pa}$ and $\eta_{p}=0.26 \mathrm{~Pa} \cdot \mathrm{s}$ on the down-curve; and $A_{t h}=39 \mathrm{kPa} / \mathrm{s}$. The other samples also strictly followed the Bingham fluid model.

We reconfirmed the assumption that the Newtonian fluid model is suitable for the predefined range of shear rates. Considering the Bingham fluid model for the lubricating layer, the range of shear rates was adjusted as follows: $\dot{\gamma}(r)=\left(\tau(r)-\tau_{y}\right) / \eta_{p}$ from Eq. (5). And applying Eq. (4) gives:

$\dot{\gamma}(r)=\frac{r}{2 \eta_{p}} \frac{\partial p}{\partial z}-\frac{\tau_{y}}{\eta_{p}}$

Replacing the viscosity with the Bingham plastic viscosity $\eta_{p}$ allowed us to keep the suggested range of shear rates only if $\tau_{y} / \eta_{p}$ was comparatively marginal. The maximum and minimum ratios of $\tau_{y} / \eta_{p}$ were $440 \mathrm{~s}^{-1}$ with $\mathrm{w} / \mathrm{cm}=0.35$ and $125 \mathrm{~s}^{-1}$ with $\mathrm{w} / \mathrm{cm}=0.60$, where the yield stress and plastic viscosity are reported in Fig. 7 and Fig. 8, respectively. Even if the maximum shear rate of interest (from Table 2) was decreased, 924-440 $=484 \mathrm{~s}^{-1}$, the trend of the flow curves remained constant for all cement paste samples (for example, see Fig. 6).

\subsection{Pressure effect}

One of the most important questions regarding the pumping process is the effect of pumping pressure on rheology. The rheological properties were determined with the down-curve to disregard the thixotropic effect. Fig. 7(a) shows a comparison of yield stress obtained in Measurement 1 (ambient pressure), where the error bar of $90 \%$ confidence interval is placed on each measurement. A sample replicate was tested at each pressure level $(0$, 10,20 , and $30 \mathrm{MPa}$ ). For example, three replicates for $w / \mathrm{cm}=0.35$ had a yield stress of $116 \pm 15 \mathrm{~Pa}$ in Measurement 1 . Those with $w /$ $\mathrm{cm}=0.40,0.45,0.50$, and 0.60 were $77 \pm 6,36 \pm 4,24 \pm 6$, and $8.6 \pm 1.2 \mathrm{~Pa}$, respectively. A comparison of the results of Measurement 1 indicated that the reproducibility of the replicated samples was acceptable. Additionally, the $w / \mathrm{cm}$ effect was clearly observed: a decrease in $w / \mathrm{cm}$ led to an increase in yield stress.

Fig. 7(b) shows the decrease in the yield stress of each sample in 


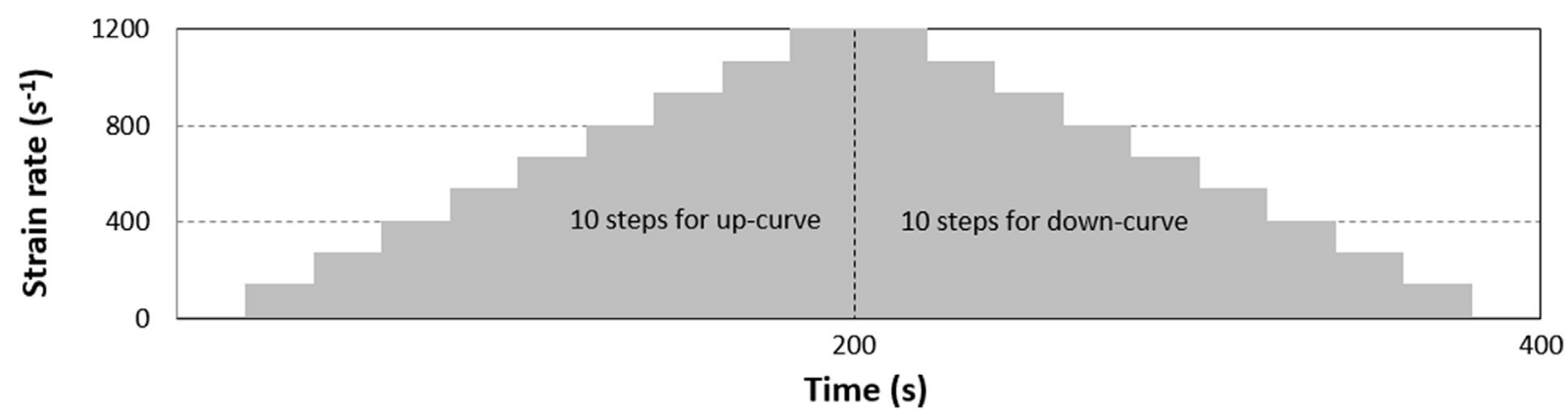

Fig. 4. Protocol of the rheological measurement.

$\begin{array}{ccccc}\text { Mixing } & \text { Placing } & \text { Pressurization } & \text { High shearing } & \text { Depressurization } \\ 3.5 \mathrm{~min} & 10 \mathrm{~min} & 15 \mathrm{~min} & 4 \mathrm{~min} & 15 \mathrm{~min}\end{array}$

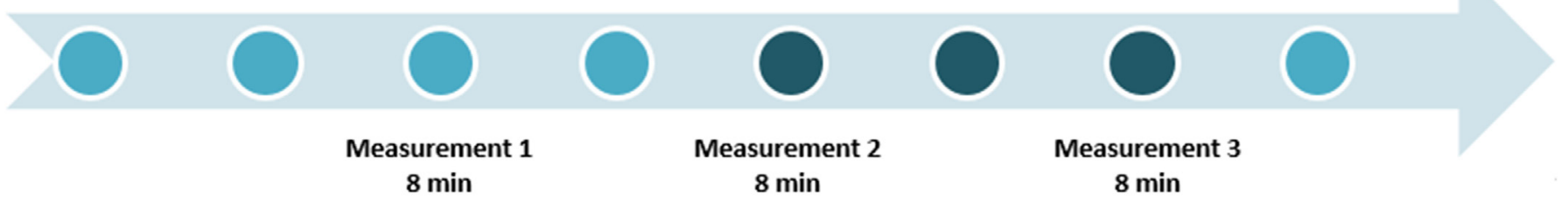

Fig. 5. Timetable for three successive measurements.

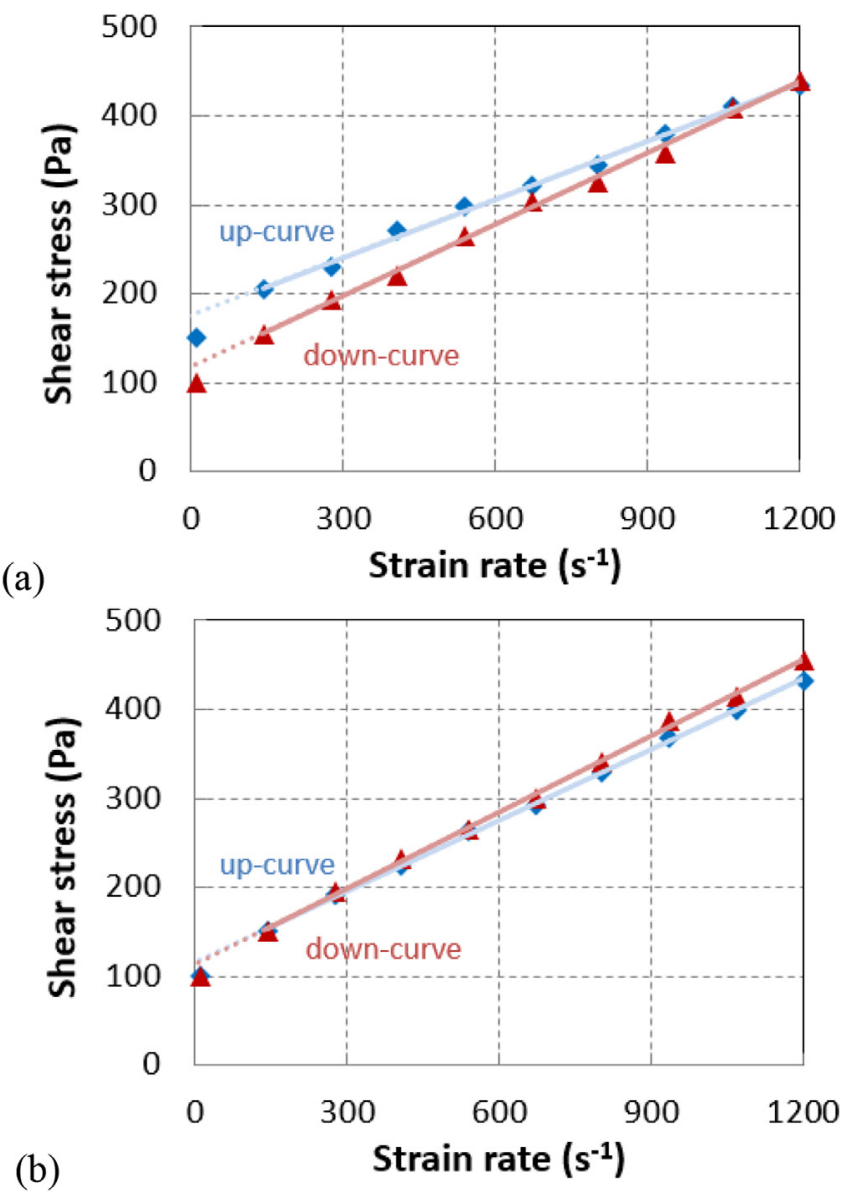

Fig. 6. Flow curve of a cement paste mixed with $w / \mathrm{cm}=0.35$ : (a) Measurement 1 and (b) Measurement 2.
Measurement 2, where the results for $w / \mathrm{cm}=0.50$ and 0.60 are not presented because they had relatively small values and changes in the yield stress. First of all, at atmospheric pressure (labeled '0' MPa in the figure), Measurement 2 considered only the effect of a $15 \mathrm{~min}$ rest period. At other pressures, this time was allocated for pressurization. At ' 0 ' MPa, the yield stress values of Measurements 1

(a)

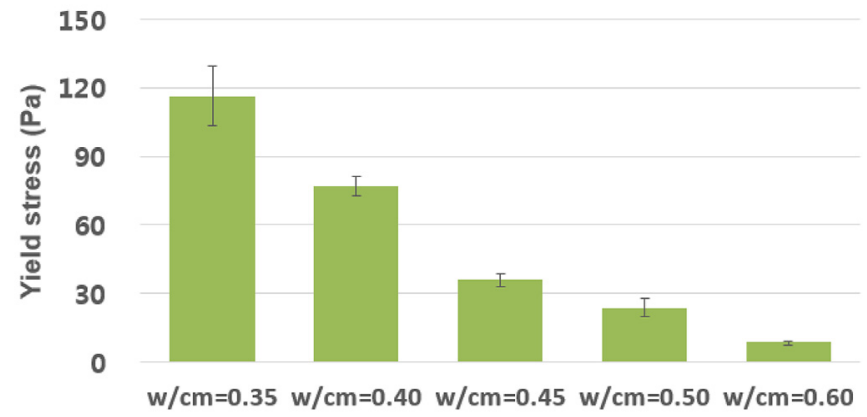

(b)

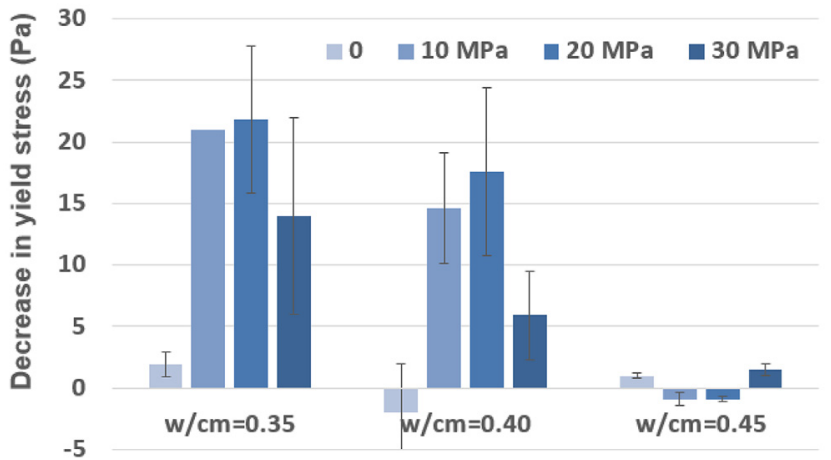

Fig. 7. Comparison of yield stress: (a) In Measurement 1 and (b) Decrease in Measurement 2 . 
and 2 were very similar. Due to the relatively high shearing and short delay, the effect of cement hydration was negligible. Under pressures greater than atmospheric, samples with a low $w / \mathrm{cm}(0.35$ and 0.40 ) had about a $15 \%$ decrease in yield stress upon pressurization (between Measurements 1 and 2), while the higher $w / \mathrm{cm}$ samples did not experience any significant pressure effects. Furthermore, the decrease in yield stress was not dependent on pressure. Flocculated cement particles are presumably broken down during pressurization, which causes the rheological properties of a mix to change during the pumping process-the smaller the solid particles in a cement suspension, the smaller the sum of their interlocking surfaces would be. The yield stress of a cement suspension is theoretically proportional to the net solidinterlocking force. A low $w / \mathrm{cm}$ sample under pumping pressure has high potential for decreased yield stress because it has a large number of flocs. Note that higher mean size were measured for lower $w / \mathrm{cm}$ cement paste [21]. This explains the $15 \%$ yield stress decrease in cement pastes with $w / \mathrm{cm}<0.40$. A decrease in yield stress (to a smaller degree) was also found in cement pastes with $w /$ $\mathrm{cm}>0.40$.

Fig. 8 shows the results of plastic viscosity. It is hard to find a trend in the change of plastic viscosity except for that the effect of pressure is more marked at low $w / \mathrm{cm}$ ratios. The variation with respect to pressure was small enough to indicate the reproducibility of replicated samples, but the variation decreased with an increase in $w / \mathrm{cm}$. This can be partially explained by considering the flocculated network as a fractal geometry. The volume can expand physically as flocs form and entrap air. Although this flocculation by cement particles causes volume expansion, it still retains selfsimilarity. In addition, a constant state of interstitial flow (here water) is manifested when the pressure effect on the plastic viscosity is minimal, but this could vary depending on the $w / \mathrm{cm}$. The interstitial fluid did not show any phase change within the levels of pressure applied and the time of interest (very early in relation to the time of setting; the initial setting time of $\mathrm{w} / \mathrm{cm}=0.35$ was $4.8 \mathrm{~h}$ approximately). Therefore, it can be said that the interstitial flow between deflocculated particles does not change with application of pressure. As a result, the yield stress and the plastic viscosity seemed to be less influenced by the pressure at a higher $w / \mathrm{cm}$.

\subsection{Rheological change during the pumping process}

We investigated the change in rheology under pumping pressure by comparing Measurements 2 and 3. The field cases introduced in Table 2 indicated that the mean duration of concrete mixes in a pipeline $350-\mathrm{m}$ long was approximately $20 \mathrm{~min}$. The timetable

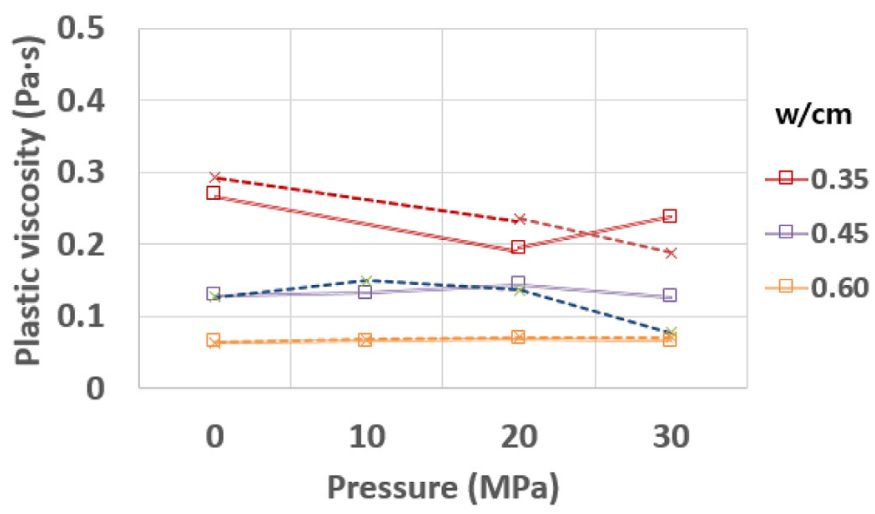

Fig. 8. Plastic viscosity of Measurement 1 (double lines with square symbols) and Measurement 2 (dashed lines with cross symbols). in Fig. 5 was designed to correspond with field conditions, and the duration of pressurization was set to $20 \mathrm{~min}$, including $4 \mathrm{~min}$ high shearing between the two measurements. Fig. 9 shows the yield stress and plastic viscosity of each sample according to the pressure level applied. First, no considerable change in the rheological properties of the cement paste was found at atmospheric pressure (' 0 ' $\mathrm{MPa}$ ). And even under pressures greater than $10 \mathrm{MPa}$, the change in yield stress was negligible between Measurements 2 and 3. In other words, no changes in the rheological properties of the cement paste were found over the investigated time duration if the pressure level was kept steady.

High shearing (up to $1,200 \mathrm{~s}^{-1}$ ) caused the microstructural evolution to cease or slow significantly, aside from that caused by the high pressure. If this finding is extended to a pumping case in field, the fluidity of the cement paste phase is expected to be constant during the concrete pumping process. Subsequently, any mixture-thickening problems such as pipe blockage and mixtureclumping during the pumping process cannot be attributed to changes in the cement paste or binding materials. Water absorption of aggregates or bleeding are expected to address these problems, but that is beyond the scope of this study.

\subsection{Change in thixotropy}

Evaluating the thixotropic parameter makes it easy to compare the up-curve and the down-curve. Fig. 10 shows the thixotropic parameters measured on cement paste samples with $w / \mathrm{cm}=0.35$ and 0.60 . For the measurements at atmospheric pressure, no additional shearing was applied during pressurization and depressurization: The sample was just left at rest in the pressure cell until the time of measurement.
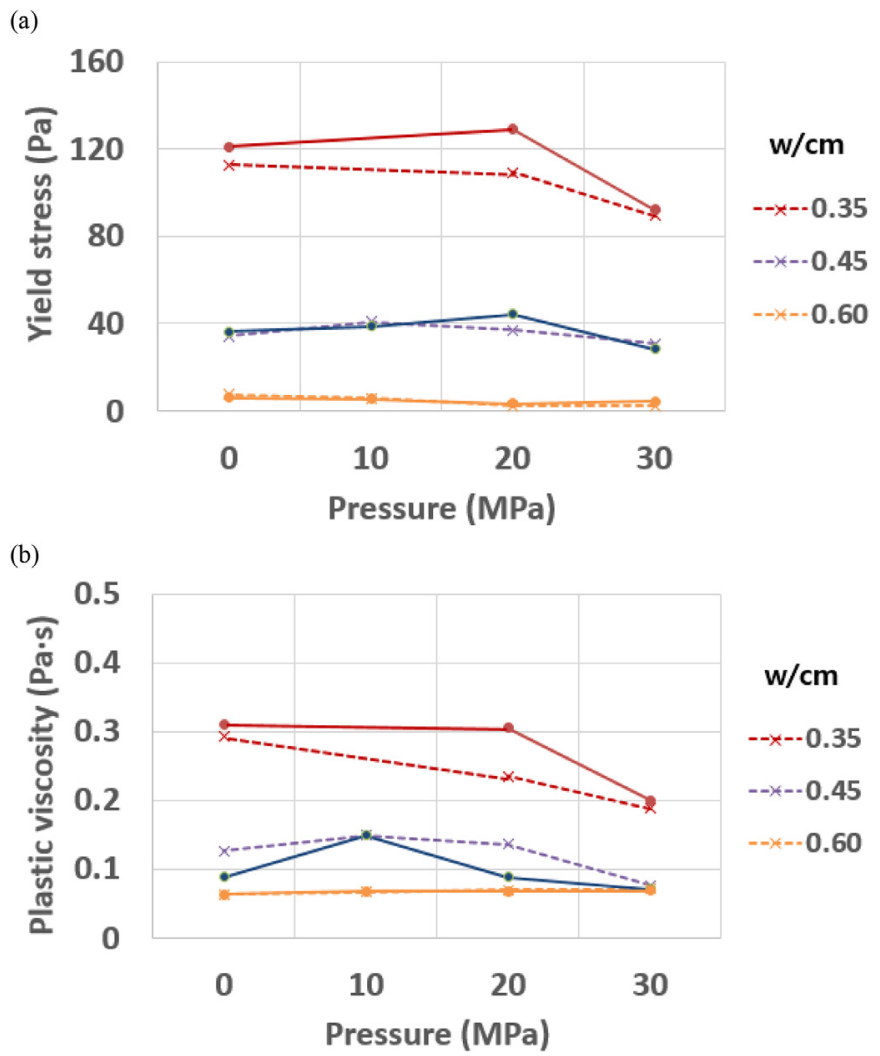

Fig. 9. Yield stress and plastic viscosity of Measurement 2 (dashed lines with cross symbols) and Measurement 3 (solid lines with circular symbols). 


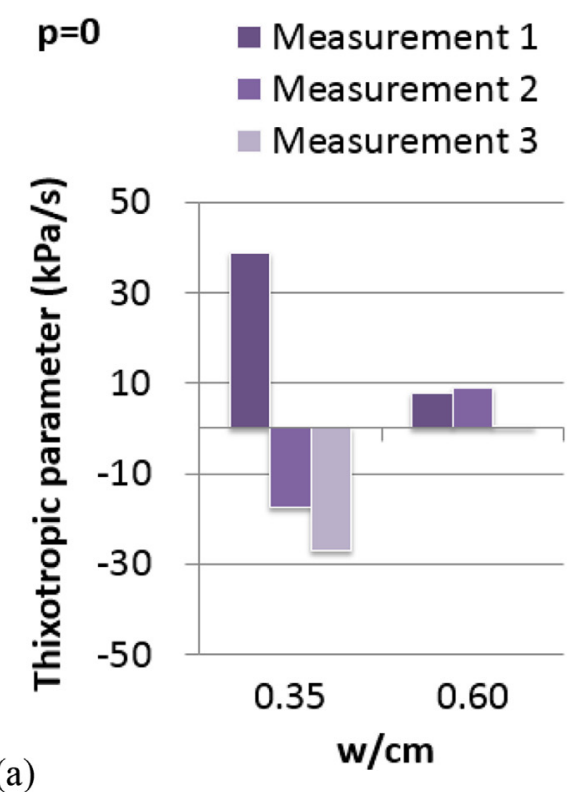

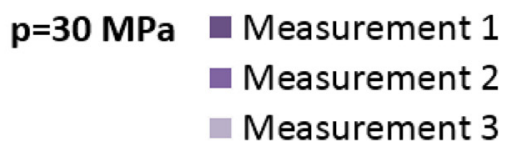

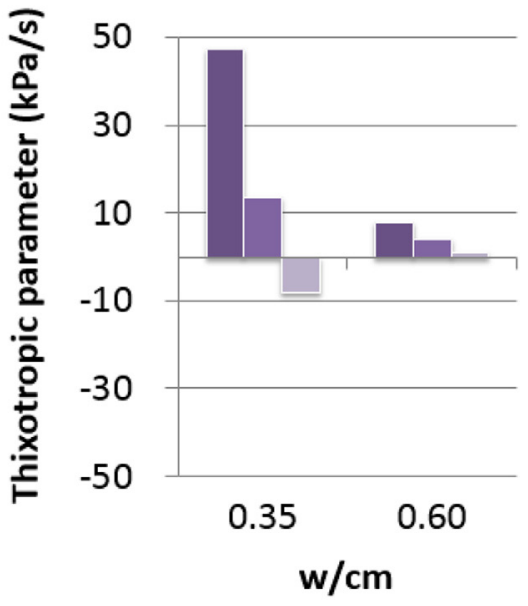

Fig. 10. Thixotropic parameters: (a) At atmospheric pressure; and (b) At $30 \mathrm{MPa}$.

Measurement 1 of each sample supports the expected effect of $w / \mathrm{cm}$ on thixotropy: a decrease in the $w / \mathrm{cm}$ led to increased thixotropy. In Measurement 2, the thixotropy of the sample decreased at both atmospheric and high pressures. In fact, the thixotropic parameter became negative at atmospheric pressure, resulting from the reversal of the up- and down-curves, which indicates rheopexy. Fig. 6(b) shows Measurement 2 of the cement paste sample, where the blue up-curve is positioned slightly below the red down-curve, but the difference between the two curves is minimal. Accelerated hydration under elevated temperatures has been reported to cause rheopexy in cement-based systems [22]. In this study, it is possible that the high shearing (up to $1,200 \mathrm{~s}^{-1}$ ) increased friction between cement particles, subsequently increasing the local temperature and accelerating hydration. Note that these effects would not fall under thixotropic phenomena because they result in irreversible changes in the structure. Nevertheless, the degree of rheopexy can be considered negligible because locally elevated temperatures and the resultant accelerated hydration would be limited to small inter-frictional areas between cement particles.

High shearing generally causes a decrease in thixotropy. Given the relatively low and sometimes negative thixotropic parameter values obtained in Measurements 2 and 3, it was assumed that during pumping thixotropic effects of the binders are negligible near the wall of the pipe (within the lubricating layer) because they would be expected to adopt high shearing during the process.

\section{Conclusions}

As a first step in investigating the mechanisms underlying the rheological changes in cementitious materials due to high pressure, this study probed neat cement pastes with $w / \mathrm{cm}$ ratios ranging from 0.35 to 0.60 . For this purpose, a rotating cylinder was equipped in a container that maintained a hydrostatic pressure. A representative range of shear rates to apply on the cement pastes were calculated from results of prior field tests on concretes; consequently a protocol of up to $1,200 \mathrm{~s}^{-1}$ was suggested with the assumption of a Newtonian fluid. The thixotropy, yield stress, and viscosity of cement paste were measured and the effect of pressure was evaluated. Results indicated approximately a $15 \%$ decrease in yield stress when a mix had a $w / \mathrm{cm}$ of less than 0.40 , while the pressure effect on the rheology was minimal at higher $w / \mathrm{cm}$ ratios. Change in the microstructure, likely in the form of deflocculation under high pressure, was the cause of the decrease in yield stress. At $w / \mathrm{cm}$ ratios higher than 0.40 , generally no considerable variation in the thixotropy and the viscosity of the cement pastes was found over a pressure range of 0 to $30 \mathrm{MPa}$. Note that at $w / \mathrm{cm}=0.35$ the thixotropy of the cement paste reversed into rheopexy after pressurization. The findings of the present study suggest that the change in the fluidity of concrete during the pumping process cannot be fully explained by the change in rheology of cement pastes in the lubricating layer. Thus more investigation is needed to explore other potential mechanisms.

\section{Acknowledgements}

This research was supported by the Basic Science Research Program through the National Research Foundation of Korea (NRF), funded by the Ministry of Science, ICT \& Future Planning (NRF2015R1A1A1A05001382).

\section{References}

[1] ACI - American Concrete Institute, Placing Concrete by Pumping Methods, American Concrete Institute, 1996, p. 25.

[2] AIJ - Architectureal Institute of Japan, Recommendations for Practices of Placing Concrete by Pumping Methods (In Japanese), Architectural Institute of Japan, 2009, p. 266.

[3] S.H. Kwon, et al., Prediction of concrete pumping: Part I-development of new tribometer for analysis of lubricating layer, ACI Mater. J. 110 (6) (2013) 647-655.

[4] S.H. Kwon, et al., Prediction of concrete pumping: Part II-analytical prediction and experimental verification, ACI Mater. J. 110 (6) (2013).

[5] R.M. Ahmed, et al., Rheology of foamed cement, Cem. Concr. Res. 39 (4) (2009) $353-361$.

[6] F. Rosquoët, et al., Experimental study of cement grout: rheological behavior and sedimentation, Cem. Concr. Res. 33 (5) (2003) 713-722.

[7] R.D. Browne, P.B. Bamforth, Tests to establish concrete pumpability, ACI J. Proc. 74 (5) (1977) 193-203.

[8] D. Kaplan, F. de Larrard, T. Sedran, Avoidance of blockages in concrete pumping process, ACI Mater. J. 102 (3) (2005) 183-191.

[9] D. Kaplan, F. de Larrard, T. Sedran, Design of concrete pumping circuit, ACI Mater. J. 102 (2) (2005).

[10] S. Jacobsen, et al., Flow conditions of fresh mortar and concrete in different 
pipes, Cem. Concr. Res. 39 (11) (2009) 997-1006.

[11] M. Choi, et al., Lubrication layer properties during concrete pumping, Cem. Concr. Res. 45 (2013) 69-78.

[12] S.D. Jo, et al., A computational approach to estimating a lubricating layer in concrete pumping, Comput. Mater. Continua 27 (3) (2011) 189-210.

[13] D. Feys, et al., Development of a tribometer to characterize lubrication layer properties of self-consolidating concrete, Cem. Concr. Compos. 54 (0) (2014) 40-52.

[17] Ouchi, M. and J. Sakue. Self-Compactability of Fresh Concrete In terms of Dispersion and Coagulation of Particles of Cement Subject to Pumping. in 3rd North American conference on the Design and Use of Self-Consolidating Concrete. 2008. Chicago, IL.

[18] J.H. Ko, et al., A study on the 1:1 full scale core wall mock-up test of high strength concrete performed by testing pumpability (in korean), J. Archit. Inst. Korea 24 (8) (2008) 203-210.

[19] J.H. Kim, H.R. Jang, H.J. Yim, Sensitivity and accuracy for rheological simulation of cement-based materials, Comput. Concr. 15 (2015) 903-919.

[20] R.P. Ferron, et al., Rheological method to evaluate structural buildup in selfconsolidating concrete cement pastes, ACI Mater. J. 104 (3) (2007) 242-250.

[21] H.J. Yim, J.H. Kim, S.P. Shah, Cement particle flocculation and breakage monitoring under Couette flow, Cem. Concr. Res. 53 (2013) 36-43.

[22] S. Al-Martini, M. Nehdi, Effect of chemical admixtures on rheology of cement paste at high temperature, J. ASTM Int. 4 (3) (2007) 1-17.

[23] S.H. Kwon, K.P. Jang, J.H. Kim, S.P. Shah, State of the art on prediction of concrete pumping, Int. J. Concr. Struct. Mater. 10 (2016) 75-85. 